\title{
Biochemical Markers of Contact with the Organic Pollutants of Urban Atmosphere in the Leaves of Woody Plants
}

\author{
Natalya Tsybulya ${ }^{1}$, Lyudmila Chindyaeva ${ }^{2}$, and Galina Dultseva ${ }^{3 *}$ \\ ${ }^{1}$ Central Siberian Botanical Garden of the Siberian Branch of the Russian Academy of Sciences \\ (CSBG SB RAS), Novosibirsk 630090, Russia \\ ${ }^{2}$ Novosibirsk State University of Architecture, Design and Arts, Novosibirsk 630099, Russia \\ ${ }^{3}$ Voevodsky Institute of Chemical Kinetics and Combustion of the Siberian Branch of the Russian \\ Academy of Sciences, Novosibirsk 630090, Russia
}

\begin{abstract}
Changes caused by contact with typical organic pollutants of urban atmosphere (formaldehyde, benzaldehyde, hydroperoxides) in the chemical composition of the leaves and shoots of woody plants are investigated. A decrease in quinic acid content and an increase in shikimic acid content were detected in the young shoots of Picea obovata Ledeb. and Picea pungens Engelm.. Investigation of changes in the chemical composition of shoots in contact with pollutants under laboratory conditions revealed that the contact with formaldehyde and hydroperoxide affects the biochemical cycle of quinic acid. High concentration of gaseous formaldehyde was found to cause an increase in the content of lactic acid in the leaves of Padus maackii (Rupr.) Kom.. In the leaves of Tilia cordata Mill. and Syringa josikaea Jacq. fil., high formaldehyde concentration caused a decrease in glycolic acid content and an increase in oxalic acid content, while after the contact with gaseous benzaldehyde this compound was detected in the leaves in rather high concentration, Analysis of the ligneous parts of plants from the territories with heavy air pollution revealed increased content of syringaldehyde, which may be a consequence of the oxidative degradation of lignin under the action of atmospheric pollutants.
\end{abstract}

\section{Introduction}

In large cities and around them, plants and adjacent forests are affected by air masses containing technogenous pollutants in high concentrations. Investigations showed that the vegetation reduces the effect of technogenous factors on the environment [1]. Promising plant species are those combining stability against foreign gases and the ability to involve the toxic gases into metabolism.

The organic components of technogenous emissions are especially dangerous for all living organisms because they form the compounds with extremely high oxidative capacity

\footnotetext{
* Corresponding author: dultseva@kinetics.nsc.ru
} 
under the action of sunlight. These oxidants are able to destroy the tissues of living organisms [2]. Many gaseous pollutants differing from each other in their chemical nature cause similar oppressive effects on the vital activities of plants [3]. Because of this, so general signs as a decrease in the activity of enzymes or changes in flavonoid content cannot serve as specific markers of the contact with the organic pollutants of urban atmosphere [4]. The goal of our work was to reveal primary biochemical markers, that is, the substances formed in the leaves of woody plants in contact with the organic pollutants in urban atmosphere, for the purpose of evaluating the capacity of species planted in cities to improve the quality of the environment. The objects of investigation were woody species planted in the Novosibirsk Scientific Center: Padus maackii (Rupr.) Kom., Tilia cordata Mill., Picea obovata Ledeb., Picea pungens Engelm., Juniperus sabina L., Syringa josikaea Jacq. fil..

\section{Materials and methods}

Air quality was monitored over the territory under investigation for two summer seasons preceding the experiment. Analysis of the concentrations of organic pollutants in the air was carried out by means of HPLC after sampling on sorption tubes filled with appropriate absorbing solutions. Acidified aqueous solution of 2,4-dinitrophenylhydrazine was used as the absorbing liquid for carbonyl compounds (aldehydes and ketones). Carboxylic acids were analyzed after sampling on the modified carbon sorbent. Aromatic compounds were sampled into a mixture of isopropanol and butanol.

Chromatographic analysis was carried out using a Milikhrom A-02 liquid chromatograph (Econova, Russia) with a column filled with the reverse-phase sorbent ProntoSil 120-5-C18 AQ, in the mode of gradient elution. Carboxylic acids were determined in the mode of ion pair chromatography. A mixture of acetonitrile (SigmaHPLC quality, $\geq 99.9 \%$,) with distilled water was used as an eluent. For ion pair chromatography, heptyl supfonate (Fisher Chemical, for Ion Pair Chromatography) (0.4\%) and trifluoroacetic acid (Fisher Chemical, 99+\%, HPLC) $(0.1 \%)$ were added into the aqueous phase. Elution was carried out at a rate of $150 \mu \mathrm{l} / \mathrm{min}$. The column was thermostated at $40{ }^{\circ} \mathrm{C}$.

Detection was carried out over optical density within the wavelength range $220-560 \mathrm{~nm}$. Identification of compounds was performed relying on the database included into the software of the chromatograph, with verification using the witness compounds: an increase in the height of chromatographic peak after the addition of the expected compound into the sample before analysis. Numerical processing of the chromatograms was carried out with the help of Alfaspektr v. 1.0.

Plant samples for investigation were collected three times during the second half of June and the first half of July in the sites chosen on the basis of the results of pollutant monitoring in the air of the Novosibirsk Scientific Center and adjacent forests. Leaves and ligneous parts were analyzed separately. Traditional extraction of the plant raw material with hot butanol turned out to be unsuitable for leaves because of the destruction of organic peroxides and formation of butyl esters, which brought distortions into the assessment of the response of plants to the contact with organic pollutants of the atmosphere. The leaves were extracted, directly after sampling, with a mixture of acetonitrile and isopropanol at room temperature. Acetonitrile, as a reagent causing denaturation of proteins, was used to stop enzymatic activity, in order to minimize the effect of sample preparation on the chemical composition of the leaves. Ligneous parts were crushed and extracted with hot butanol.

For experiments in laboratory chamber, gaseous formaldehyde was generated by means of pyrolysis of paraform (Chemically Pure grade, Reakhim, Russia). Formaldehyde 
concentration in the chamber was measured by means of HPLC in the form of the derivative of 2,4-dinnitriphenylhydrazine. The vapors of tert-butyl hydroperoxide and benzaldehyde were admitted into the chamber in nitrogen flow. The concentrations of these compounds were measured by means of HPLC.

\section{Results and discussion}

In the sites of B type, formaldehyde concentration in the air did not exceed 0.2 MPC (the maximum permissible concentration), while benzaldehyde was below detection limit. A comparison between the chromatograms of the extracts from the leaves of the same species collected from sites A and B showed that the differences in the number of peaks and in peak intensities are observed for all the studied plant species except Juniperus sabina.. The presence of organic atmospheric pollutants was found to affect the young shoots of Picea obovata and Picea pungens. The needles of trees from site A contained a lower amount of quinic acid (the concentration was lower by $32-41 \%$, with the average value $35.3 \%$ over 10 measurements). The concentration of shikimic acid was higher by $23 \%$ as average than in the needles of trees from B type sites. It is known that the transformation of quinic acid into shikimic acid in plants proceeds through a reversible equilibrium with 3-dehydroquinic acid, but the attempts to reveal its presence in the plants from A type sites failed, though this compound was clearly detected in the plants from the sites of B type. This fact allowed us to suppose that the organic atmospheric pollutants possessing oxidative properties affect the equilibrium between quinic and 3-dehydroquinic acids and thus lead to a sharp decrease in the steady concentration of the latter. To prove this hypothesis, we placed the young shoots of $P$. obovata from site B into the chamber with the vapor of model organic compounds imitating urban pollutants. These model compounds were formaldehyde, benzaldehyde, and tert-butyl hydroperoxide. Analysis results showed that after the contact with gaseous formaldehyde $\left(75 \mu \mathrm{g} / \mathrm{m}^{3}\right)$ for $30 \mathrm{~min}$ the concentration of quinic acid decreased by $4.3 \pm 2.2 \%$, and after the contact with tert-butyl hydroperoxide for the same time interval this concentration decreased by $2.6 \pm 0.9 \%$. The contact with gaseous benzaldehyde did not cause any changes in the concentration of quinic acid. After the contact of the shoots with a mixture of formaldehyde and tert-butyl hydroperoxide in the same concentrations for the same interval of time, we detected a decrease in the concentration of quinic acid by $14.5 \pm 3.8 \%$. This result allows us to assume that the observed decrease in the concentration of quinic acid in the needles of plants from the sites with substantial air pollution may be a sign of long-term action of both formaldehyde and hydroperoxides, which are the products of its atmospheric transformation.

An increase in the content of lactic (oxypropionic) acid $\mathrm{CH}_{3} \mathrm{CH}(\mathrm{OH}) \mathrm{COOH}$ by $22 \%$ as average was detected in the leaves of Padus maackii from the sites of A type in comparison with its content in the plants from B type sites. Experiments with the leaves cut off the trees and placed in the chamber showed that the contact with hydroperoxide has no effect on the concentration of lactic acid, while the contact with gaseous formaldehyde causes a substantial increase in its content. Results of the measurement of lactic acid content in the leaves of Padus maackii are presented in Fig. 1. 


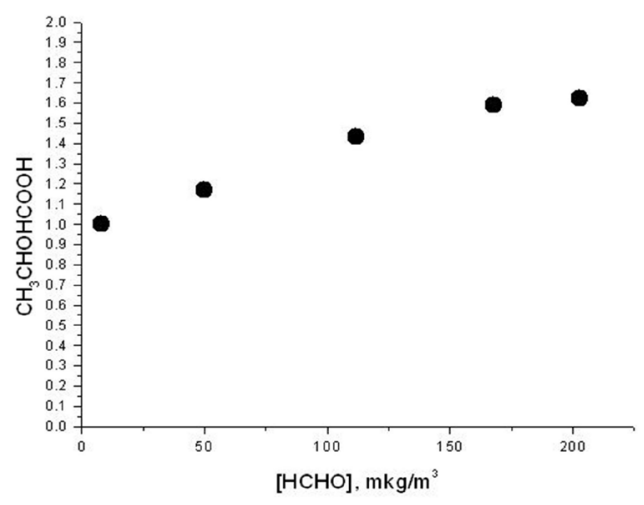

Fig. 1. Relative concentration of lactic acid in the leaves of Padus maackii changing with an increase in the concentration of gaseous formaldehyde in the chamber. Lactic acid content in the intact leaves is taken as 1 .

In the leaves of Tilia cordata and Syringa josikaea from the sites of A type, we detected a decrease in the concentration of glycolic (oxyacetic) acid $\mathrm{HOCH}_{2} \mathrm{COOH}$ (by $9 \%$ in tilia and by $18 \%$ in syringa as average) and an increase in the content of oxalic acid HOOC$\mathrm{COOH}$ (by 18 and $23 \%$, respectively). Scattering of the results turned out to be substantial (up to $\pm 7 \%$ ). Then we measured the concentrations of these acids in the leaves after contact with formaldehyde, benzaldehyde, and tert-butyl hydroperoxide in the experimental chamber. Contact with hydroperoxide and benzaldehyde did not cause any changes in acid content. Benzaldehyde was detected in the extracts of the leaves of both species. Contact with formaldehyde caused a substantial increase in the content of oxalic acid and a decrease in the content of glycolic acid as shown in Fig. 2.

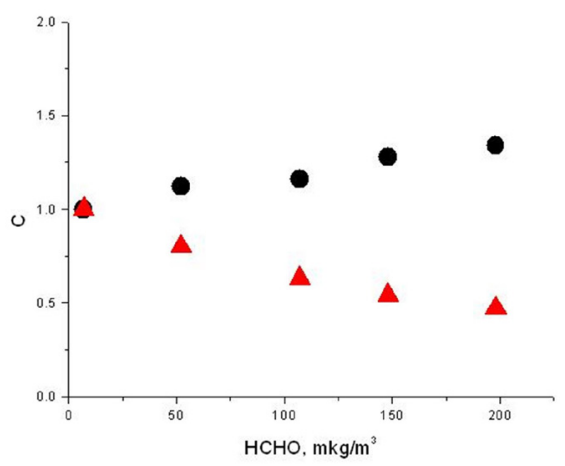

Fig. 2. Changes in the relative concentrations (C) of glycolic acid (triangles) and oxalic acid (circles) in the leaves of Syringa josikaea after contact with gaseous formaldehyde in the experimental chamber.

An increased content of syringaldehyde was detected in the extracts of ligneous parts of plants from the sites of A type (by $22 \%$ as average) in comparison with the level of this compound in the extracts of plants from the sites of B type. Larger amount of syringaldehyde in the trees growing under heavy air pollution may be explained by the oxidative degradation of lignin under the action of atmospheric pollutants. However, experimental studies in the chamber did not reveal such changes. A possible reason may be insufficient time of contact with pollutants. So, we suppose that the accumulation of 
syringaldehyde in the ligneous parts of woody plants may serve as an indicator of the oxidative degradation of lignin due to long-term contact with atmospheric pollutants.

\section{Conclusions}

The compounds revealed in the present work in the leaves of several plant species may serve as the markers of the contact with the organic pollutants of urban atmosphere. One of these markers is quinic acid. Its content in the young shoots of Picea obovata and Picea pungens decreases as a result of contact with formaldehyde and hydroperoxide.

An increase in lactic acid content was detected in the leaves of Padus maackii under the action of increased concentrations of formaldehyde. In the leaves of Tilia cordata and Syringa josikaea, contact with increased formaldehyde concentration causes an increase in the content of oxalic acid and a decrease in the content of glycolic acid, which is the evidence of formaldehyde involvement into metabolism. Accumulation of syringaldehyde in ligneous parts is a non-specific sign of long-term contact with the organic pollutants of the atmosphere. A possible reason of the accumulation of syringaldehyde in woody plants may be lignin destruction under the action of organic atmospheric pollutants.

\section{References}

1. G.F. Gheorghe, B. Ion. The effects of air pollutions on vegetation and the role of vegetation in reducing atmospheric pollution, in The Impact of Air Pollution on Health, Economy, Environment and Agricultural Sources. (InTech, Rijeka, 2011)

2. W.M. Dugger, J. Koukol, R.L. Palmer, Journ. Air Pollut. Control Association, 16, 467$471(1966)$

3. S. Shyam, K. Nath, D. Singh. Res. Environ. Life Sci., 1, 65-68 (2008)

4. C.M. Furlan, A. Salatino. Atm. Pollut. Res., 1, 30-35 (2010) 